\title{
Vinculación sustentable con los graduados a través de una red social y alcance de ésta
}

\author{
Marcela Agostini, Marcela Trapé, Graciela Spretz, Laura París, Guillermo Weisburd
}

Objetivos. Determinar qué utilidad brindan las redes sociales para sustentar el vínculo con los graduados de una carrera de medicina, indagar su inserción en las residencias/concurrencias y explorar cuántos graduados están involucrados en actividades académicas institucionales.

Sujetos y métodos. Estudio observacional, descriptivo y transversal. Se estableció una relación con los graduados a través de una red social, para lo cual se creó un grupo en el mes de mayo de 2011 con una denominación que los identificaba y se les envío la suscripción para unirse al grupo. A medida que iban aceptando, los mismos graduados invitaban a sus compañeros a unirse. Se utilizó como herramienta de trabajo una encuesta semiestructurada online para conocer la realidad de los graduados, que se administró desde julio hasta octubre de 2011. La población fueron todos los graduados de la carrera de medicina, sede Rosario, de una universidad de gestión privada, desde diciembre de 2002 hasta agosto de 2011.

Resultados. De los 374 graduados se estableció contacto con 269. De ellos, respondieron la encuesta 220 graduados. Con respecto al nivel de formación de posgrado, 179 graduados continuaron su formación académica: 131 ingresaron al régimen de residencias, 10 a concurrencias, 27 a posgrados y 11 a la práctica médica integral. Respecto a las especialidades elegidas, se halló un gran predominio por las orientadas al manejo clínico del paciente y, en segundo lugar, las de un abordaje quirúrgico.

Conclusión. Las redes sociales permitieron a corto plazo hacer sustentable el vínculo con los graduados e indagar cuál es su inserción en los sistemas de residencias-concurrencias y posgrados en el área de la salud.

Palabras clave. Graduados. Red social. Residencias.

\section{Sustainable relationship with the graduates through a social network and scope of the same}

Aims. To determine the usefulness of social networks to sustain the link with the graduates, to investigate the inclusion in nursing/turnout, and to explore how many graduates are involved in academic institutions.

Subjects and methods. We performed an observational, descriptive and transversal. A relationship with graduates through a social network. A group in the month of May 2011 with a designation identifying them and sending them a subscription to join the group. As they are accepting the group was forming, they invited their fellow graduates to join. It was used a working tool semistructured questionnaire sent via online to monitor the reality of the graduates: surveys were sent from July 2011 to October 2011. The people were all graduates of the medical career of a university-based Rosario privately owned understood from December 2002 to August 2011.

Results. Of the 374 graduates contact was established with 269. Of the 269 respondents contacted 220 graduates. Regarding the level of 179 postgraduate training graduates continued their education: 131 graduates entered the residence status, 10 occurrences, 27 postgraduates, and 11 comprehensive medical practice. With respect to the chosen specialties found a high prevalence for those facing clinical management and secondly those of a surgical approach.

Conclusion. Social networks allow us in the short term make sustainable links with our graduates and monitor their integration into systems, concurrency and graduate residences in the area of health.

Key words. Graduates. Medical residences. Social network.
Universidad Abierta Interamericana. Rosario, Argentina.

Correspondencia:

Profa. Marcela Agostini. Universidad Abierta Interamericana. Lagos, 944. Rosario, Argentina.

E-mail:

marcela.agostini@vaneduc.edu.ar

Conflicto de intereses: No declarado.

Competing interests: None declared.

(c) 2013 FEM 


\section{Introducción}

Las redes sociales permiten una gestión muy eficiente cuando hay implicado un gran número de alumnos y profesores. Cuanto mayor sea el número de miembros de una red social, mayor será su productividad. Una red social con 500 miembros será mucho más efectiva que una con 100. Por debajo de 100 alumnos, las redes sociales pierden su eficacia y no merece la pena su utilización [1].

La innovación se ha tornado una inquietud creciente en países desarrollados y emergentes. Se implementan políticas y estrategias sobre ciencia y tecnología, dado que esta área es el motor fundamental de las innovaciones. Los países más desarrollados -y un gran número de países emergentes, como Brasil, India y China- han implementado políticas nacionales dirigidas a estimular la capacidad de innovación de sus industrias y empresas. El reclamo de 'Internet para todos' es actualmente moneda corriente. Sus defensores argumentan que las tecnologías de la información y la comunicación (TIC), tarde o temprano, satisfarán el ideal democrático del acceso universal al conocimiento y el logro de sociedades genuinamente democráticas, basadas en la transparencia, la solidaridad, la participación de los ciudadanos y los derechos humanos. Un punto de vista menos utópico plantea que estas tecnologías pueden utilizarse para imponer hegemonías que tenderían a beneficiar a las elites ya existentes, agravar las brechas sociales, acentuar las relaciones de inequidad y dominación y, en consecuencia, debilitar las economías pequeñas o marginales y a los países más frágiles [2-4].

El seguimiento de egresados como una acción investigadora tiene relación con el análisis y reformulación curricular, al tiempo que se articula con los requerimientos nacionales que determinan la calidad educativa en torno a la proyección social de los programas de una institución, la coherencia de su proyecto educativo con sus programas y el énfasis en la innovación y el desarrollo sustentado en los procesos de investigación. En este sentido, el seguimiento de egresados es un asunto de vital importancia, ya que el desempeño profesional y personal de los egresados permite establecer indicadores con respecto a la calidad y eficiencia de las instituciones [5].

Las universidades son instituciones de referencia en sus respectivos entornos sociales, tanto por la labor que realizan generando graduados como por el importante papel social que desempeñan. No obstante, la mayoría de las universidades no están desarrollando políticas eficaces que fomenten mantener y estrechar los lazos de relación con sus pro- pios egresados. El presente trabajo plantea la conveniencia de utilizar las redes sociales como instrumento de fidelización de los egresados universitarios, que tendría como consecuencia inmediata la mejora de la formación permanente de los graduados universitarios y, también, conocer su inserción en residencias, concurrencias y posgrados.

\section{Sujetos y métodos}

Estudio observacional, descriptivo y transversal. Se estableció una relación con los graduados a través de una red social, Facebook. Se creó un grupo en el mes de mayo de 2011 con una denominación que los identificaba y se les envío la suscripción para unirse al grupo. A medida que iban aceptando se fue conformando el grupo, los mismos graduados invitaban a sus compañeros a unirse. Se utilizó como herramienta de trabajo una encuesta semiestructurada vía online para conocer la realidad de los graduados. Las encuestas se enviaron desde julio hasta octubre de 2011.

La población fueron todos los graduados de la carrera de medicina, sede Rosario, de una universidad de gestión privada, entre diciembre de 2002 y agosto de 2011.

Las categorías de análisis abordadas fueron:

- Identificación de los egresados.

- Nivel de formación de posgrado: residencias-concurrencias, posgrado, práctica médica integral (PMI) o ninguna.

- Lugar de formación: gestión pública o privada.

- Especialidades elegidas.

- Inserción en actividades académicas (docencia-extensión e investigación) en nuestra universidad.

\section{Resultados}

De los 374 graduados se estableció contacto con 269. No aceptaron todavía la solicitud enviada 53; no tenían red social 41, constatado vía correo, y un graduado falleció. De los 269 contactados, respondieron la encuesta 220 graduados.

Con respecto al nivel de formación de posgrado, 179 graduados continuaron su formación académica de la siguiente manera: 131 ingresaron al régimen de residencias, 10 a concurrencias, 27 a posgrados y 11 a PMI.

Con respecto al lugar de formación, 92 fueron en un centro privado y 87 en hospitales públicos. Se detallan en la tabla I los países, provincias y ciudades donde desarrollaron su formación. No contestaron este ítems 13 graduados. 
Tabla I. Provincias y ciudades del país donde realizaron su formación de residencias, concurrencias posgrados y prácticas medicas integrales.

\begin{tabular}{|c|c|c|c|}
\hline & Provincia & Ciudad & $n$ \\
\hline \multirow[t]{20}{*}{ Argentina } & Santa Fe & Rosario & 71 \\
\hline & & Venado Tuerto & 10 \\
\hline & & Santa Fe & 8 \\
\hline & & Granadero Baigorria & 2 \\
\hline & & Villa Constitución & 1 \\
\hline & & Reconquista & 1 \\
\hline & Buenos Aires & Capital Federal & 32 \\
\hline & & Mar del Plata & 10 \\
\hline & & San Nicolás & 3 \\
\hline & & La Plata & 2 \\
\hline & & Junín & 2 \\
\hline & & Pergamino & 2 \\
\hline & & Tigre & 1 \\
\hline & & Bahía Blanca & 1 \\
\hline & & Zárate & 1 \\
\hline & & Pilar & 1 \\
\hline & Entre Ríos & Paraná & 4 \\
\hline & Tierra del Fuego & Ushuaia & 2 \\
\hline & Córdoba & San Francisco & 1 \\
\hline & Neuquén & Neuquén & 1 \\
\hline Brasil & & & 9 \\
\hline España & & Barcelona & 1 \\
\hline
\end{tabular}

Con respecto a las especialidades elegidas se halló un gran predominio por las orientadas al manejo clínico del paciente y, en segundo lugar, las de un abordaje quirúrgico. Se eligieron 26 especialidades diferentes (Tabla II).

Se incorporaron a tareas académicas 32 graduados, de los cuales 18 ingresaron como auxiliares en actividades docentes, 10 en actividades de extensión
Tabla II. Especialidades elegidas.

\begin{tabular}{|c|c|}
\hline & $n$ \\
\hline Clínica médica & 22 \\
\hline Diagnóstico por imágenes & 15 \\
\hline Psiquiatría & 14 \\
\hline Cirugía general & 14 \\
\hline Oftalmología & 13 \\
\hline Traumatología & 11 \\
\hline Tocoginecología & 11 \\
\hline Obstetricia & 9 \\
\hline Cardiología & 9 \\
\hline Pediatría & 9 \\
\hline Medicina laboral & 6 \\
\hline Patólogo & 2 \\
\hline Neurocirugía & 2 \\
\hline Dermatología & 2 \\
\hline Fisioterapia & 2 \\
\hline Terapeuta & 2 \\
\hline Urología & 2 \\
\hline Anestesista & 1 \\
\hline Generalista & 1 \\
\hline Neurología & 1 \\
\hline Nutricionista & 1 \\
\hline Otorrinolaringología & 1 \\
\hline Infectología & 1 \\
\hline Medicina del deporte & 1 \\
\hline
\end{tabular}

pertenecientes al 'Programa del Aula de Medicina en la Comunidad' y 4 como becarios en actividades de investigación.

\section{Discusión}

$\mathrm{Al}$ igual que en el trabajo realizado por Uribe-Tirado 
y Echavarría-Ramírez [6] en Colombia, hemos hallado un beneficio en la utilización de este recurso -redes sociales- para lograr sostener una relación activa con los graduados y controlar su carrera de formación. Coincidimos en que más que una herramienta para interactuar con amigos y familiares, es ideal para crear comunidad y establecer contactos comerciales y laborales. El análisis realizado en su trabajo confirma la tendencia cultural y tecnológica de las personas de buscar cada vez más la 'convergencia', definida como la capacidad de adaptación y el uso de una herramienta tecnológica para una variedad de propósitos y el empleo de Facebook como medio de interacción social y para la interacción profesional y laboral, por lo que asumen funciones diferentes.

El proceso de seguimiento a egresados se constituye en una herramienta que propicia el acercamiento de la institución de educación superior a los egresados, conociendo de ellos sus expectativas, la correspondencia de su desempeño laboral con las competencias de formación adquiridas y las perspectivas de formación de posgrado [5]. Coincidimos con la utilidad de esta herramienta para brindar información y mantener un diálogo permanente y continuo, en un mismo lenguaje virtual, lo cual nos acerca aún más a nuestros graduados.

Es inherente al proceso de calidad institucional el seguimiento de sus graduados, dado que son éstos quienes representan a la universidad y sus programas en el contexto [5,6]. Es fundamental poder dar cuenta de nuestros graduados porque son nuestro mejor ejemplo del trabajo realizado durante su etapa de formación académica de grado.

En 2009, Galli [7,8] advierte que Argentina, con una población estimada de 39 millones de habitantes, tiene un sistema de educación universitaria integrado por 94 instituciones, un 50\% de gestión estatal y el otro $50 \%$ de gestión privada. También hace referencia al sistema de residencias médicas, considerado como el mejor sistema de formación para la práctica de una especialidad y también para la práctica de la medicina general. En la formación de los especialistas existe una gran variedad de situaciones y superposiciones. La Ley de Educación Superior establece que sólo las universidades pueden otorgar títulos de posgrado y la Ley de Ejercicio Profesional, que regula la práctica de la medicina, autoriza a 'anunciarse como especialista' a quien haya completado una residencia o haya cumplido cinco años de asistencia en un servicio de la especialidad. En nuestro estudio se observa una tendencia a ingresar en sistemas de residencias, tanto en centros públicos como privados $[9,10]$.
Brissón y Galli [11] tienen como propósito identificar cuáles son los temas de la educación médica que se han debatido en Argentina en los últimos veinte años, cuáles son los que ocupan la agenda actual y cuáles son los emergentes, así como establecer algunas relaciones con el movimiento global en ese campo. Los temas planteados se agrupan en cinco grandes categorías, siendo una de ellas la 'evaluación de la calidad de los egresados'. El resultado que se obtiene expresa la preocupación por la relación entre lo que se enseña y 'el médico que el país necesita', pero no se comunican investigaciones al respecto. Ante la ausencia de estudios de seguimiento de egresados, nuestro trabajo intenta dar respuestas a este interrogante. Cabe destacar la importancia de haber logrado el uso de esta herramienta a través de la web 2.0, ya que mediante métodos tradicionales, como teléfonos o correo, no se podía sustentar en el tiempo debido a la migración de los graduados a nuevas ciudades para iniciar un nuevo ciclo de formación y también por la necesidad de tener que actualizarnos tecnológicamente.

El grupo de Figueroa [12] pone de manifiesto cómo integraron estrategias constructivistas junto a la tecnología que brinda el web 2.0, de manera que pudieron identificar las características de los estudiantes, construir espacios dinámicos donde integraron blogs, wikis y podcasts en el escenario de las clases, lograron combinar estrategias de retención con el uso de esta vía y utilizaron las redes sociales para estar comunicados. Si bien es un trabajo realizado con alumnos de pregrado en una universidad de Puerto Rico, resulta totalmente posible trasladar esta experiencia con la realizada por nosotros con graduados gracias a las TIC. Es un desafío para nosotros poder incorporar a nuestros graduados todas las otras modalidades que este docente empleó con sus alumnos y poder contar los resultados de esta nueva experiencia.

Las redes sociales nos permitieron a corto plazo hacer sustentable el vínculo con nuestros graduados y controlar su inserción en los sistemas de residencias-concurrencias y posgrados en el área de la salud. Queda de manifiesto que el uso de la web 2.0 no puede demorarse mas y debe aplicarse en todas las funciones y modalidades que ofrece.

Bibliografía

1. Cabero J, Pérez F. Estrategias didácticas para la red. URL: http://tecnologiaedu.us.es/tics3. [16.10.2004].

2. Cabero J. Las TIC y la universidad. Sevilla: MAD; 2002.

3. Cabero J. Las nuevas tecnologías en la actividad universitaria. Píxel-Bit, Revista de Medios y Educación 2003; 20: 81-100.

4. Espuny C, González J, Lleixà M, Gisbert M. Actitudes y expectativas del uso educativo de las redes sociales en los 
alumnos universitarios. In: El impacto de las redes sociales en la enseñanza y el aprendizaje. Revista de Universidad y Sociedad del Conocimiento (RUSC) 2011; 8: 171-85.

5. Marulanda-Galvis J, Ortiz-Botero E, Moratto-Vásquez N, Patricia A, Rojas A. Caracterización de egresados de la universidad en las cohortes de 2003, 2005, 2007 y momento '0.' Revista CES Psicología 2010; 3: 50-63.

6. Uribe-Tirado A, Echavarría-Ramírez A. Facebook como red de profesionales de bibliotecología, documentación y archivística en Iberoamérica. El Profesional de la Información 2008; 17: 670-6.

7. XVI Conferencia Panamericana de Educación Médica. Autoevaluación y acreditación en tiempos de cambio. Educ Med 2003; 6 (Supl 1): S7-27.
8. Galli A. Situación actual de la educación médica en Argentina. Educ Med 2009; 12: 3-5.

9. Ministerio de Salud. Resolución 1342/2007. URL: http:// www.puntoprofesional.com.ar/p/meduc/meduc_1314_07.htm. [10.01.2008].

10. Brissón ME, Galli A, Morera MI. Estándares para la acreditación de carreras de medicina: el proceso de revisión. Revista Argentina de Educación Médica 2011; 4: 76-86.

11. Brissón ME, Galli A. Conferencia Argentina de Educación Médica: agendas, aportes y temas eergentes. Educ Med 2005; 8: 38-47.

12. Figueroa J. Usando la web 2.0 como estrategia para la retención estudiantil. URL: http://hdl.handle.net/123456789/2943. 